\title{
Rationally cubic connected manifolds II
}

\author{
Gianluca Occhetta and Valentina Paterno
}

\begin{abstract}
We study smooth complex projective polarized varieties $(X, H)$ of dimension $n \geq 2$ which admit a dominating family $V$ of rational curves of $H$-degree 3 , such that two general points of $X$ may be joined by a curve parametrized by $V$ and which do not admit a covering family of lines (i.e., rational curves of $H$-degree one). We prove that such manifolds are obtained from RCC manifolds of Picard number one by blow-ups along smooth centers.

If we further assume that $X$ is a Fano manifold, we obtain a stronger result, classifying all Fano RCC manifolds of Picard number $\rho_{X} \geq 3$.
\end{abstract}

\section{Introduction}

In our recent paper [15], inspired by the classification of conic-connected manifolds given in [10], we started the study of rationally cubic connected manifolds (RCC-manifolds, for short), i.e., smooth complex projective polarized varieties $(X, H)$ of dimension $n \geq 2$ which are rationally connected by rational curves of degree 3 with respect to a fixed ample line bundle $H$.

In [15] we considered manifolds covered by lines (i.e., rational curves of degree one with respect to $H$ ), proving that the Picard number of such manifolds is at most three and that if equality holds then the manifold has an adjunction scroll structure over a smooth variety.

In the present paper we will complete our task by dealing with RCC-manifolds not covered by lines. Our main result shows that such manifolds are obtained by RCC-manifolds of Picard number one by blow-ups along smooth centers. More precisely we have the following:

Theorem 1.1. Let $(X, H)$ be an $R C C$-manifold with respect to $V$, not covered by lines. Then there exists a polarized manifold $\left(X^{\prime}, H^{\prime}\right)$ of Picard number one not covered by lines and a contraction $\varphi_{\Sigma}: X \rightarrow X^{\prime}$ expressing $X$ as a blow-up of $X^{\prime}$ along disjoint smooth irreducible centers $T_{i}$ with exceptional divisors $E_{i}$ such

Mathematics Subject Classification (2010): Primary 14M22; Secondary 14J40, 14J45, 14 E30. Keywords: Rationally connected manifolds, rational curves, Fano manifolds. 
that $H \simeq \varphi_{\Sigma}^{*} H^{\prime}-\sum E_{i}$. The pair $\left(X^{\prime}, H^{\prime}\right)$ is $R C C$ with respect to $V^{\prime}$, the family of deformations of the image of a general curve parametrized by $V$.

Moreover either $\left(X^{\prime}, H^{\prime}\right) \simeq\left(\mathbb{P}^{n}, \mathcal{O}_{\mathbb{P}}(3)\right)$ or $\operatorname{Pic}\left(X^{\prime}\right)=\left\langle H^{\prime}\right\rangle$ and $-K_{X^{\prime}}=\frac{n+1}{3} H^{\prime}$. In the latter case we have $\operatorname{dim} T_{i}<(n+1) / 3$ for every $i$.

First of all we deal with the case of RCC-surfaces, giving a complete classification of them in Section 3, then we move to the general case.

The main idea is the following: if the Picard number of $X$ is greater than one, then for every point $x \in X$ the cubics parametrized by $V$ passing through $x$ must degenerate into reducible cycles whose components are not numerically proportional to $V$. Clearly these degenerations can be into cycles consisting either of three lines or of a line and a conic. Since we are assuming that $X$ is not covered by lines, the latter happens for a general point, and moreover the irreducible component through the general point is the conic, so for each possible degeneration we get a dominating family of conics and a family of lines.

It turns out that the loci of the families of lines arising in this way are divisors, which we will call divisors of $V$-lines; the main point of the proof is to show that these divisors are (disjoint) exceptional divisors of smooth blow-ups and that they do not meet a general cubic, so that, after blowing them down, we still have a RCC-manifold.

The hardest case is that of manifolds of Picard number two, which is treated in Section 5, while the general one is settled in Section 6.

If we further assume that the manifold is Fano, the results are much stronger and for $\rho_{X} \geq 3$ we have a complete classification:

Theorem 1.2. Let $(X, H)$ be a polarized Fano manifold of dimension $n>2$ and $\rho_{X} \geq 3$. Suppose that $X$ is $R C C$ with respect to a family $V$ and doesn't admit a covering family of lines. Then $\rho_{X}=3, X$ has a contraction $\pi: X \rightarrow \mathbb{P}^{n}$ which is the blow-up of $\mathbb{P}^{n}$ along two disjoint centers $T_{1}$ and $T_{2}$ which can be:

(1) two linear spaces $\Lambda_{1}, \Lambda_{2}$ such that

$$
\Lambda_{1} \cap \Lambda_{2}=\emptyset, \quad \operatorname{dim} \Lambda_{1}+\operatorname{dim} \Lambda_{2}=n-2,
$$

(2) a linear space $\Lambda_{1}$ and a smooth quadric $Q_{1} \subset \Lambda_{2} \simeq \mathbb{P}^{\operatorname{dim} Q_{1}+1}$ such that

$$
\Lambda_{1} \cap \Lambda_{2}=\emptyset, \quad \operatorname{dim} Q_{1} \geq \frac{n}{2}-1, \quad \operatorname{dim} \Lambda_{1}+\operatorname{dim} Q_{1}=n-2,
$$

(3) two smooth quadrics $Q_{1} \subset \Lambda_{1} \simeq \mathbb{P}^{\frac{n}{2}}$ and $Q_{2} \subset \Lambda_{2} \simeq \mathbb{P}^{\frac{n}{2}}$ such that

$$
Q_{1} \cap Q_{2}=\emptyset, \quad \operatorname{dim} \Lambda_{1} \cap \Lambda_{2}=0, \quad \operatorname{dim} Q_{1}=\operatorname{dim} Q_{2}=\frac{n}{2}-1,
$$

and, denoting by $E_{1}$ and $E_{2}$ the exceptional divisors, $H \simeq \pi^{*} \mathcal{O}_{\mathbb{P}^{n}}(3)-E_{1}-E_{2}$.

Section 7 is devoted to the proof of this theorem, and to some other considerations on RCC Fano manifolds of Picard number two.

In the last section we examine in detail the manifolds appearing in Theorem 1.2, describing their cones of curves, their contractions, and their families of rational curves. 


\section{Background material}

We gather in this section the basic definitions and results regarding families of rational curves and Mori theory that we are going to use.

\subsection{Families of rational curves and of rational 1-cycles}

Definition 2.1. A family of rational curves $V$ on $X$ is an irreducible component of the scheme $\operatorname{Ratcurves}^{n}(X)$ (see Definition II.2.11 in [13]).

Given a rational curve we will call a family of deformations of that curve any irreducible component of $\operatorname{Ratcurves}^{n}(X)$ containing the point parametrizing the curve.

We define $\operatorname{Locus}(V)$ to be the set of points of $X$ through which there is a curve among those parametrized by $V$; we say that $V$ is a covering family if $\operatorname{Locus}(V)=$ $X$ and that $V$ is a dominating family if $\overline{\operatorname{Locus}(V)}=X$.

By abuse of notation, given a line bundle $H \in \operatorname{Pic}(X)$, we will denote by $H \cdot V$ the intersection number $H \cdot B$, with $B$ any curve among those parametrized by $V$. We will say that $V$ is unsplit if it is proper; clearly, an unsplit dominating family is covering.

We denote by $V_{x}$ the subscheme of $V$ parametrizing rational curves passing through a point $x$ and by $\operatorname{Locus}\left(V_{x}\right)$ the set of points of $X$ through which there is a curve among those parametrized by $V_{x}$. If, for a general point $x \in \operatorname{Locus}(V)$, $V_{x}$ is proper, then we will say that the family is locally unsplit. Moreover, we say that $V$ is generically unsplit if, through a general $x \in \operatorname{Locus}(V)$ and a general $y \in \operatorname{Locus}\left(V_{x}\right)$ there is a finite number of curves parametrized by $V$.

Definition 2.2. We define a Chow family of rational 1 -cycles $\mathcal{W}$ to be an irreducible component of Chow $(X)$ parametrizing rational and connected 1-cycles. If $V$ is a family of rational curves, the closure of the image of $V$ in $\operatorname{Chow}(X)$, denoted by $\mathcal{V}$, is called the Chow family associated with $V$. If $V$ is proper, i.e., if the family is unsplit, then $V$ is the normalization of the associated Chow family $\mathcal{V}$.

Definition 2.3. Let $V$ be a family of rational curves and let $\mathcal{V}$ be the associated Chow family. We say that $V$ (and also $\mathcal{V}$ ) is quasi-unsplit if every component of any reducible cycle parametrized by $\mathcal{V}$ has numerical class proportional to the numerical class of a curve parametrized by $V$.

Definition 2.4. We say that $k$ quasi-unsplit families $V^{1}, \ldots, V^{k}$ are numerically independent if in $\mathrm{N}_{1}(X)$ we have $\operatorname{dim}\left\langle\left[V^{1}\right], \ldots,\left[V^{k}\right]\right\rangle=k$.

For special families of rational curves we have useful dimensional estimates. The basic one is the following:

Proposition 2.5. ([13, Corollary IV.2.6]) Let $V$ be a family of rational curves on $X$ and $x \in \operatorname{Locus}(V)$ a point such that every component of $V_{x}$ is proper. Then

(a) $\operatorname{dim} \operatorname{Locus}(V)+\operatorname{dim} \operatorname{Locus}\left(V_{x}\right) \geq \operatorname{dim} X-K_{X} \cdot V-1$;

(b) every irreducible component of $\operatorname{Locus}\left(V_{x}\right)$ has dimension $\geq-K_{X} \cdot V-1$. 
Definition 2.6. Let $V^{1}, \ldots, V^{k}$ be families of rational curves on $X$ and $Z \subset X$. We define Locus $\left(V^{1}\right)_{Z}$ to be the set of points $x \in X$ such that there exists a curve $C$ among those parametrized by $V^{1}$ with $C \cap Z \neq \emptyset$ and $x \in C$. We inductively define $\operatorname{Locus}\left(V^{1}, \ldots, V^{k}\right)_{Z}:=\operatorname{Locus}\left(V^{k}\right)_{\operatorname{Locus}\left(V^{1}, \ldots, V^{k-1}\right)_{Z}}$.

Notation: If $\Gamma$ is a 1-cycle, then we will denote by $[\Gamma]$ its numerical equivalence class in $\mathrm{N}_{1}(X)$; if $V$ is a family of rational curves, we will denote by $[V]$ the numerical equivalence class of any curve among those parametrized by $V$. A proper family will always be denoted by a calligraphic letter.

If $Z \subset X$, we will denote by $\mathrm{N}_{1}(Z, X) \subseteq \mathrm{N}_{1}(X)$ the vector subspace generated by numerical classes of curves of $X$ contained in $Z$; moreover, we will denote by $\mathrm{NE}(Z, X) \subseteq \mathrm{NE}(X)$ the subcone generated by numerical classes of curves of $X$ contained in $Z$. We will denote by $\langle\ldots\rangle$ the linear span.

We will use some properties of $\operatorname{Locus}(V)_{Z}$, summarized in the following

Lemma 2.7 (Section 5 of [1], proof of Lemma 1.4.5 in [4]). Let $Z \subset X$ be an irreducible closed subset and $\mathcal{V}$ an unsplit family. Then every curve contained in $\operatorname{Locus}(\mathcal{V})_{Z}$ is numerically equivalent to a linear combination with rational coefficients

$$
\lambda C_{Z}+\mu C_{\mathcal{V}}
$$

where $C_{Z}$ is a curve in $Z, C_{\mathcal{V}}$ is a curve among those parametrized by $\mathcal{V}$ and $\lambda \geq 0$. If moreover curves contained in $Z$ are numerically independent from curves in $\mathcal{V}$ and $Z \cap \operatorname{Locus}(\mathcal{V}) \neq \emptyset$ then

$$
\operatorname{dim} \operatorname{Locus}(\mathcal{V})_{Z} \geq \operatorname{dim} Z-K_{X} \cdot \mathcal{V}-1
$$

We will also need the following lemma, which is based on Proposition II.4.19 in $[13]$ :

Lemma 2.8. Let $Y \subset X$ be a closed subset, and let $\mathcal{V}$ be a Chow family of rational 1-cycles. Then every curve contained in $\operatorname{Locus}(\mathcal{V})_{Y}$ is numerically equivalent to a linear combination with rational coefficients of a curve contained in $Y$ and of irreducible components of cycles parametrized by $\mathcal{V}$ which meet $Y$.

\subsection{Contractions and fibrations}

Definition 2.9. Let $X$ be a manifold such that $K_{X}$ is not nef. Denote by $\overline{\mathrm{NE}}(X) \subset$ $\mathrm{N}_{1}(X)$ the closure of the cone of effective 1-cycles in the $\mathbb{R}$-vector space of 1-cycles modulo numerical equivalence, and by $\overline{\mathrm{NE}}(X)_{K_{X}<0}$ the set $\left\{z \in \mathrm{N}_{1}(X): K_{X}\right.$. $z<0\}$. An extremal face is a face $\sigma$ of $\overline{\mathrm{NE}}(X)$, associated with some nef line bundle $L$, contained in the negative part of the cone with respect to $K_{X}$, i.e., $\sigma=\overline{\mathrm{NE}}(X) \cap L^{\perp} \subset \overline{\mathrm{NE}}(X)_{K_{X}<0}$; an extremal face of dimension one is called an extremal ray.

With an extremal face $\sigma \subset \mathrm{NE}(X)$ is associated a morphism with connected fibers $\varphi_{\sigma}: X \rightarrow Z$ onto a normal variety, that contracts the curves whose numerical class is in $\sigma ; \varphi_{\sigma}$ is called an extremal contraction or a Fano-Mori contraction, while 
a Cartier divisor $H$ such that $H=\varphi_{\sigma}^{*} A$ for an ample divisor $A$ on $Z$ is called a supporting divisor of the map $\varphi_{\sigma}$ (or of the face $\sigma$ ). We denote by $\operatorname{Exc}\left(\varphi_{\sigma}\right):=$ $\left\{x \in X \mid \operatorname{dim} \varphi_{\sigma}^{-1}\left(\varphi_{\sigma}(x)\right)>0\right\}$ the exceptional locus of $\varphi_{\sigma}$.

An extremal contraction associated with an extremal ray is called an elementary contraction; an elementary contraction is said to be of fiber type if $\operatorname{dim} X>\operatorname{dim} Z$, otherwise the contraction is birational. Moreover, if the codimension of the exceptional locus of an elementary birational contraction is equal to one, then the contraction is called divisorial; otherwise it is called small.

Proposition 2.5, in the case $\mathcal{V}$ is the unsplit family of deformations of a minimal extremal rational curve, gives the fiber locus inequality:

Proposition 2.10 ([9], [17]). Let $\varphi$ be a Fano-Mori contraction of $X$ and let $E=\operatorname{Exc}(\varphi)$ be its exceptional locus; let $S$ be an irreducible component of a (nontrivial) fiber of $\varphi$. Then

$$
\operatorname{dim} E+\operatorname{dim} S \geq \operatorname{dim} X+l-1,
$$

where $l=\min \left\{-K_{X} \cdot C \mid C\right.$ is a rational curve in $\left.S\right\}$. If $\varphi$ is the contraction of a ray $R$, then $l(R):=l$ is called the length of the ray.

The next theorem, which will be frequently used, gives us conditions to ensure that a birational contraction is a smooth blow-up:

Theorem 2.11 (Theorem 4.1 (iii) in [2]). Let $\varphi: X \rightarrow Z$ be an extremal contraction of a smooth variety $X$. Assume that $\varphi$ is birational and supported by $K_{X}+r H$, with $H$ a $\varphi$-ample line bundle on $X$, and that, for each non-trivial fiber $F$ of $\varphi$ we have $\operatorname{dim} F=r$. Then $Z$ is smooth and $\varphi$ is a blow down of a smooth divisor $E \subset X$ to a smooth subvariety of $Z$.

\section{Preliminaries}

Definition 3.1. Let $(X, H)$ be a polarized manifold of dimension $n$; if there exists a dominating family $V$ of rational curves such that $H \cdot V=3$ and through two general points of $X$ there is a curve parametrized by $V$ we will say that $X$ is Rationally Cubic Connected-RCC for short- with respect to $V$.

In Proposition 4.1 of [15] we proved the following result concerning RCC manifolds of Picard number one:

Proposition 3.2. Let $(X, H)$ be a $R C C$-manifold with respect to a family $V$. Then,

1) there exists $x \in X$ such that $V_{x}$ is proper if and only if $(X, H) \simeq\left(\mathbb{P}^{n}, \mathcal{O}(3)\right)$;

2) there exists $x \in X$ such that $V_{x}$ is quasi-unsplit but not proper if and only if $X$ is a Fano manifold of Picard number one and index $r(X) \geq \frac{n+1}{3}$ with fundamental divisor $H$. 
As a consequence, if the Picard number of $X$ is greater than one, through a general point there exists at least one reducible cycle parametrized by the Chow family $\mathcal{V}$ whose components are not all numerically proportional to $V$.

Since $H \cdot V=3$, such a cycle in $\mathcal{V}$ can have two or three irreducible rational components; we will call a component of $H$-degree one a line and a component of $H$-degree two a conic.

Throughout the present paper, after dealing with the case of surfaces in the next subsection, we will assume that $X$ does not admit a covering family of lines; this assumption yields that every dominating family of conics is locally unsplit. Moreover, from Formula (1), Corollary 5.6 and Formula (4) in [15] we have that $V$ is generically unsplit and so

$$
-K_{X} \cdot V=n+1
$$

\subsection{RCC surfaces}

We will now give a complete classification of RCC-surfaces. As a consequence, we will see that Theorem 1.1 holds for $n=2$.

Proposition 3.3. Let $(S, H)$ be a polarized surface, which is $R C C$ with respect to a family of rational curves $V$. Then $(S, H)$ and $V$ are one of the following:

1. $\left(\mathbb{P}^{2}, \mathcal{O}_{\mathbb{P}^{2}}(3)\right)$, the family of lines in $\mathbb{P}^{2}$;

2. $\left(\mathbb{P}^{2}, \mathcal{O}_{\mathbb{P}^{2}}(1)\right)$, the family of rational plane cubics;

3. $\left(\mathbb{Q}^{2}, \mathcal{O}_{\mathbb{Q}^{2}}(1,2)\left(\right.\right.$ or $\left.\left.\mathcal{O}_{\mathbb{Q}^{2}}(2,1)\right)\right)$, the family of curves of type $(1,1)$;

4. $\left(\mathbb{Q}^{2}, \mathcal{O}_{\mathbb{Q}^{2}}(1,1)\right)$, the family of curves of type $(2,1)($ or $(1,2))$;

5. $\left(\mathbb{F}_{1}, C_{0}+3 f\right)$, the family of curves of type $C_{0}+f$;

6. $\left(S_{k},-K_{S_{k}}\right)$ with $S_{k}$ a blow-up of $\mathbb{P}^{2}$ in $k$ general points, with $k=1, \ldots, 8$, the family of strict transforms of lines in $\mathbb{P}^{2}$.

Proof. By the first part of Proposition 3.2, if $V_{x}$ is proper for some $x \in S$ then $(S, H) \simeq\left(\mathbb{P}^{2}, \mathcal{O}_{\mathbb{P}^{2}}(3)\right)$, while, by the second part, if $V_{x}$ is quasi-unsplit for some $x \in S$ then $(S, H) \simeq\left(\mathbb{P}^{2}, \mathcal{O}_{\mathbb{P}^{2}}(1)\right)$.

We can assume from now on that the Picard number of $S$ is at least two.

If $V$ is not generically unsplit then either $(S, H)=\left(\mathbb{Q}^{2}, \mathcal{O}_{\mathbb{Q}^{2}}(1,2)\right)$ (and curves of $V$ are curves of type $(1,1))$ or through every pair of points of $S$ there is a reducible cycle in $V$ consisting of three lines by Proposition 5.5 in [15]. In this last case $S$ admits two dominating families of lines, hence $(S, H) \simeq\left(\mathbb{Q}^{2}, \mathcal{O}(1,1)\right)$ and $V$ is, up to exchanging the rulings, the family of curves of type $(2,1)$.

We are thus left with the case of $V$ generically unsplit. By formula (3.1) we then have $-K_{S} \cdot V=3$.

We consider first the case in which $S$ admits a covering family of lines $\mathcal{L}$. Recalling that $\rho_{S} \geq 2$, we have that $S$ is a ruled surface $\mathbb{F}_{e}=\mathbb{P}_{\mathbb{P}^{1}}(\mathcal{O}(-e) \oplus \mathcal{O})$, and the lines are the fibers of the projection to $\mathbb{P}^{1}$. 
Denote by $C_{0}$ a minimal section and by $f$ a fiber; since the fibers are lines with respect to $H$ we can write $H \equiv C_{0}+h f$. Let $B \equiv a C_{0}+b f$ be a curve parametrized by $V$. By the genus formula we get

$$
1=B^{2}=a(2 b-a e)
$$

hence $a=1$ and $e=2 b-1$. Since $B$ is an effective curve, this is possible just for $b=e=1$. Now, from $H \cdot B=3$, we get $h=3$.

Finally we treat the case of a surface not covered by lines.

Consider the set $\mathcal{B}^{\prime}=\left\{\left(\mathcal{L}^{i}, C^{i}\right)\right\}$ of pairs of families $\left(\mathcal{L}^{i}, C^{i}\right)$ such that through a general point $x \in S$ there is a reducible cycle $\ell+\gamma$ belonging to $\mathcal{V}$, with $\ell$ and $\gamma$ parametrized respectively by $\mathcal{L}^{i}$ and $C^{i}$.

The families of conics are locally unsplit and dominating, hence $-K_{S} \cdot C^{i}=2$ for every $i$; this implies that $-K_{S} \cdot \mathcal{L}^{i}=1$. The numerical classes of $\mathcal{L}^{i}$ and $C^{i}$ are a system of generators for $N_{1}(S)$ (by Lemma 2.8), and $K_{S}+H$ is trivial on each of them.

It follows that $H$ and $-K_{S}$ are numerically equivalent; being $S$ rational they are also linearly equivalent. In particular $-K_{S}$ is ample, and $S$ is a del Pezzo surface. We are now assuming that the Picard number of $S$ is greater than one, and that $-K_{S} \cdot V=3$, so $S$ is not a projective space or a quadric.

\section{Divisors of $V$-lines}

Having settled the case of surfaces in Proposition 3.3, we will assume from now on that $n=\operatorname{dim} X \geq 3$.

Consider the set $\mathcal{B}^{\prime}=\left\{\left(\mathcal{L}^{i}, C^{i}\right)\right\}$ of pairs of families $\left(\mathcal{L}^{i}, C^{i}\right)$ such that through a general point $x \in X$ there is a reducible cycle $\ell+\gamma$, parametrized by $\mathcal{V}$, with $\ell$ and $\gamma$ parametrized respectively by $\mathcal{L}^{i}$ and $C^{i}$.

Let us consider two pairs $\left(\mathcal{L}^{i}, C^{i}\right)$ and $\left(\mathcal{L}^{j}, C^{j}\right)$ such that $\left[\mathcal{L}^{i}\right] \neq\left[\mathcal{L}^{j}\right]$. Since no family of lines is covering, by the generality of $x$ all the families of conics are dominating and locally unsplit; therefore $\operatorname{dim} \operatorname{Locus}\left(C^{i}\right)_{x} \cap \operatorname{Locus}\left(C^{j}\right)_{x}=0$ for every $i \neq j$. It follows that

$$
-K_{X} \cdot\left(C^{i}+C^{j}\right) \leq \operatorname{dim} \operatorname{Locus}\left(C^{i}\right)_{x}+\operatorname{Locus}\left(C^{j}\right)_{x}+2 \leq n+2,
$$

so, recalling that $-K_{X} \cdot\left(C^{i}+\mathcal{L}^{i}\right)=-K_{X} \cdot V=n+1$, we also have

$$
-K_{X} \cdot\left(\mathcal{L}^{i}+\mathcal{L}^{j}\right) \geq n .
$$

Let now $\mathcal{B}=\left\{\left(\mathcal{L}^{i}, C^{i}\right)\right\}_{i=1}^{k}$ be a maximal set of pairs in $\mathcal{B}^{\prime}$ with the property that $[V],\left[\mathcal{L}^{1}\right], \ldots,\left[\mathcal{L}^{k}\right]$ are numerically independent. Denote by $\Pi_{i}$ the twodimensional vector subspace of $\mathrm{N}_{1}(X)$ spanned by $[V]$ and $\left[\mathcal{L}^{i}\right]$. By Lemma 2.8,

$$
\mathrm{N}_{1}(X)=\left\langle[V],\left[\mathcal{L}^{1}\right],\left[C^{1}\right], \ldots,\left[\mathcal{L}^{k}\right],\left[C^{k}\right]\right\rangle=\left\langle[V],\left[\mathcal{L}^{1}\right],\left[\mathcal{L}^{2}\right], \ldots,\left[\mathcal{L}^{k}\right]\right\rangle,
$$

hence the Picard number of $X$ is $k+1$. 
For every $i=1, \ldots, k$ denote by $E_{i}$ the set Locus $\left(C^{i}, \mathcal{L}^{i}\right)_{x}$; by Lemma 2.7 and by Proposition 2.5 it has dimension $\operatorname{dim} E_{i} \geq n-1$; since $E_{i} \subset \operatorname{Locus}\left(\mathcal{L}^{i}\right)$, the inclusion is an equality and $E_{i}$ is an irreducible divisor.

We will call the divisor $E_{i}$ the divisor of $V$-lines associated with the pair $\left(\mathcal{L}^{i}, C^{i}\right)$. We will distinguish the divisors of $V$-lines in the following way:

1. $E_{i}$ is of the first kind if $-K_{X} \cdot \mathcal{L}^{i}=n-1$;

2. $E_{i}$ is of the second kind if $-K_{X} \cdot \mathcal{L}^{i}=1$;

3. $E_{i}$ is of the third kind in all the other cases.

The next lemma gives the description of the relative space of 1-cycles of the divisors of $V$-lines.

Lemma 4.1. Let $E_{i}$ be a divisor of $V$-lines, associated with a pair $\left(\mathcal{L}^{i}, C^{i}\right)$. If $E_{i}$ is of the first kind then $\mathrm{N}_{1}\left(E_{i}, X\right)=\left\langle\left[\mathcal{L}^{i}\right]\right\rangle$. In the other cases $\mathrm{N}_{1}\left(E_{i}, X\right)=\left\langle\left[C^{i}\right],\left[\mathcal{L}^{i}\right]\right\rangle$ and $\left[\mathcal{L}^{i}\right]$ is extremal in $\mathrm{NE}\left(E_{i}, X\right)$.

Proof. If $E_{i}$ is of the first kind, then $E_{i}=\operatorname{Locus}\left(\mathcal{L}^{i}\right)_{x}$ for any $x \in \operatorname{Locus}\left(\mathcal{L}^{i}\right)$, while if $E_{i}$ is either of the second or of the third kind then $E_{i}=\operatorname{Locus}\left(C^{i}, \mathcal{L}^{i}\right)_{x}$ for a general $x \in X$. The statement now follows from Lemma 2.7.

As a consequence, we can prove that these divisors are disjoint:

Lemma 4.2. Let $E_{i}$ for $i=1, \ldots, k$ be divisors of $V$-lines associated with pairs in $\mathcal{B}$. Then the $E_{i}$ 's are pairwise disjoint. Moreover, if $E_{k}$ is of the third kind then $E_{i} \cdot \mathcal{L}^{k}=E_{i} \cdot C^{k}=0$ for every $i \neq k$.

Proof. Since we are assuming that $n \geq 3$, if two divisors met, their intersection would be positive dimensional. Therefore, by the description of the relative space of cycles $\mathrm{N}_{1}\left(E_{i}, X\right)$, it is clear that any divisor of the first kind is disjoint from any other divisor. Moreover, if a divisor of the second kind exists, then, by equation (4.1), all the other divisors are of the first kind.

We will now show that, if $E_{k}$ is of the third kind then $E_{i} \cdot \mathcal{L}^{k}=E_{i} \cdot C^{k}=0$ for every $i \neq k$. This implies also that two divisors of the third kind are disjoint.

Both $\operatorname{dim} \operatorname{Locus}\left(C^{k}\right)_{x}$ and $\operatorname{dim} \operatorname{Locus}\left(\mathcal{L}^{k}\right)_{x}$ are greater than one, so if $E_{i} \cdot C^{k}>0$ (respectively $E_{i} \cdot \mathcal{L}^{k}>0$ ) then $E_{i}$ would contain a curve whose numerical class is proportional to $\left[C^{k}\right]$ (resp. $\left[\mathcal{L}^{k}\right]$ ), a contradiction, since neither $\left[C^{k}\right]$ nor $\left[\mathcal{L}^{k}\right]$ is contained in $\mathrm{N}_{1}\left(E_{i}, X\right)$.

Theorem 1.1 will follow if we prove that all the divisors of $V$-lines have intersection number zero with $V$. In fact we have the following:

Proposition 4.3. Let $\mathcal{F}=\left\{E_{1}, \ldots, E_{k}\right\}$ be a collection of pairwise disjoint divisors of $V$-lines such that $E_{i} \cdot V=0$ for every $i=1, \ldots, k$.

Then there exist a polarized manifold $\left(X^{\prime}, H^{\prime}\right)$ not covered by lines and a contraction $\varphi_{\sigma}: X \rightarrow X^{\prime}$ expressing $X$ as a blow-up of $X^{\prime}$ along $k$ disjoint centers $T_{i}$, with exceptional divisors $E_{1}, \ldots, E_{k}$ and such that $H=\varphi_{\sigma}^{*} H^{\prime}-\sum E_{i}$.

Moreover $\left(X^{\prime}, H^{\prime}\right)$ is $R C C$ with respect to $V^{\prime}$, the family of deformations of the image of a general curve parametrized by $V$. 
Proof. The effective divisor $E_{i}$ cannot be trivial on the whole $\mathrm{NE}(X)$; since it vanishes on $[V]$, which lies in the interior of $\mathrm{NE}(X)$, it must be negative on some effective curve $B$. This curve is therefore contained in $E_{i}$.

By Lemma 4.1, the numerical class of $B$ is contained in the two-dimensional vector subspace of $\mathrm{N}_{1}(X)$ spanned by $\left[C^{i}\right]$ and $\left[\mathcal{L}^{i}\right]$. Since $E_{i} \cdot C^{i} \geq 0$, being $C^{i}$ a dominating family, and $E_{i} \cdot V=0$ we have $E_{i} \cdot \mathcal{L}^{i}<0$.

Consider the divisor $H_{i}=-\left(E_{i} \cdot \mathcal{L}^{i}\right) H+E_{i}$. We will show that this divisor is nef and trivial only on $R^{i}=\mathbb{R}_{+}\left[\mathcal{L}^{i}\right]$. Assume that, for some curve $B$ we have $H_{i} \cdot B \leq 0$. This implies $E_{i} \cdot B<0$, so $B \subset E_{i}$, hence $[B] \subset \mathrm{NE}\left(E_{i}, X\right)$.

Recalling that $\left[\mathcal{L}^{i}\right]$ is extremal in $\mathrm{NE}\left(E_{i}, X\right)$, it is clear that for every curve whose numerical class is in $\mathrm{NE}\left(E_{i}, X\right) \subset \Pi_{i}$ the intersection number with $H_{i}$ is nonnegative, and it is zero if and only if $[B] \in R^{i}$; hence $H_{i}$ is nef and $R^{i}$ is an extremal ray of $\mathrm{NE}(X)$.

Denote by $\varphi_{i}$ the contraction associated with $R^{i}$. Since $\operatorname{Locus}\left(\mathcal{L}^{i}\right)=E_{i}$ and $E_{i} \cdot R^{i}<0$ then $\operatorname{Exc}\left(\varphi_{i}\right)=E_{i}$. Moreover, since $E_{i}=\operatorname{Locus}\left(\mathcal{L}^{i}\right)_{\operatorname{Locus}\left(C^{i}\right)_{x}}$ for a general $x \in X$, any fiber $F_{i}$ of $\varphi_{i}$ meets $\operatorname{Locus}\left(C^{i}\right)_{x}$, hence

$$
n \geq \operatorname{dim} F_{i}+\operatorname{dim} \operatorname{Locus}\left(C^{i}\right)_{x} \geq-K_{X} \cdot \mathcal{L}^{i}-K_{X} \cdot C^{i}-1=n .
$$

Equality must then hold. In particular for any fiber of $\varphi_{i}$ we have $\operatorname{dim} F_{i}=$ $-K_{X} \cdot \mathcal{L}^{i}$. Thus, $\varphi_{i}$ is a smooth blow-up by Theorem 2.11. Notice that from this it follows that $E_{i} \cdot \mathcal{L}^{i}=-1$.

Consider now the divisor $H+\sum E_{i}$. Arguing as we did for $H_{i}$, we prove that it is nef and it vanishes only on curves whose numerical classes belong to one of the $R^{i}$ 's. Therefore there is a $k$-dimensional face $\sigma$ of $\mathrm{NE}(X)$ generated by the $R^{i}$ 's and the associated contraction $\varphi_{\sigma}: X \rightarrow X^{\prime}$ contracts exactly the curves whose numerical class belongs to $R^{i}$ for some $i$. Since the $E_{i}$ 's are disjoint, $\varphi_{\sigma}$ is the blow-up of $X^{\prime}$ along smooth disjoint centers $T_{i}$ 's.

Let $V^{\prime}$ be a family of deformations of the image of a general curve parametrized by $V$. Clearly, through two general points of $X^{\prime}$ there is a curve parametrized by $V^{\prime}$. The divisor $H+\sum E_{i}$ is nef and supports the face contracted by $\varphi_{\sigma}$, hence there exists an ample divisor $H^{\prime}$ on $X^{\prime}$ such that $\varphi_{\sigma}^{*} H^{\prime}=H+\sum E_{i}$. From the projection formula we get $H^{\prime} \cdot V^{\prime}=3$.

Assume by contradiction that $X^{\prime}$ is covered by lines, i.e., there exists a dominating family of rational curves $\mathcal{L}^{\prime}$ of degree one with respect to $H^{\prime}$. The family $\mathcal{L}$ of deformations of the strict transform of a general line $\ell^{\prime}$ parametrized by $\mathcal{L}^{\prime}$ will be a covering family of lines for $X$; in fact, being general, $\ell^{\prime}$ is disjoint from $T_{i}$ for every $i$, hence $H \cdot \mathcal{L}=1$.

\section{Manifolds of Picard number two}

In this section we are going to prove the first part of Theorem 1.1 under the assumption that the Picard number of $X$ is two. This is the hardest case and represents a crucial step in the proof. 
Let $\mathcal{B}^{\prime}=\left\{\left(\mathcal{L}^{i}, C^{i}\right)\right\}$ be as in the previous section; as we saw, to each pair in $\mathcal{B}^{\prime}$ is associated a divisor $E_{i}=\operatorname{Locus}\left(\mathcal{L}^{i}\right)$; we need to show that one of them is the exceptional divisor of a smooth blow-up and does not meet a general curve of $V$.

We deal first with a particular case, namely the case in which a divisor of $V$-lines of the second kind exists.

Proposition 5.1. Let $(X, H)$ be an $R C C$-manifold with respect to $V$, not covered by lines and of Picard number two. Assume that there exists a divisor $E$ of $V$-lines which is of the second kind. Then there exists a contraction $\varphi: X \rightarrow \mathbb{P}^{n}$ expressing $X$ as a blow-up of $\mathbb{P}^{n}$ along a codimension two linear subspace or along a codimension two smooth quadric. Moreover $H=\varphi^{*} \mathcal{O}_{\mathbb{P}^{n}}(3)-E$ and $V$ is the family of deformations of the strict transform of a general line in $\mathbb{P}^{n}$.

Proof. Let $(\mathcal{L}, C)$ be the pair in $\mathcal{B}^{\prime}$ whose associated divisor of $V$-lines is $E$. The two cases appearing in the statement differ by the position in $\mathrm{NE}(X)$ of the numerical class $[C]$. Assume first that $[C]$ spans an extremal ray of $\mathrm{NE}(X)$.

The associated contraction $\psi: X \rightarrow B$ is then of fiber type with fibers of dimension $n-1$. In fact a fiber $F$ contains $\operatorname{Locus}\left(C_{x}\right)$ for every $x \in F$ and, for a general $x \in X$ we have $\operatorname{dim} \operatorname{Locus}\left(C_{x}\right) \geq n-1$, since $E$ is a divisor of the second kind. A general fiber of $\psi$ is a projective space by Theorem 3.6 in [12]. Since the contraction is elementary, by standard arguments we get that $X$ is a projective bundle over $B$; since $X$ is rationally connected we have that $B$ is rational, and thus $X=\mathbb{P}_{\mathbb{P}^{1}}(\mathcal{E})$ with $\mathcal{E}=\oplus \mathcal{O}\left(a_{i}\right)$ and $0=a_{0} \leq a_{1} \leq \cdots \leq a_{n}$.

The family $C$ is the family of lines in the fibers of $\psi$. Recalling that $H \cdot C=2$ and denoting by $\xi_{\mathcal{E}}$ the tautological line bundle of $\mathcal{E}$, we can write $H=2 \xi_{\mathcal{E}}+\psi^{*} \mathcal{O}(b)$ for some $b$. The ampleness of $H$ yields $b \geq 1$; equality holds, since $H \cdot \mathcal{L}=1$. Moreover from the last formula we get that a curve of $\mathcal{L}$ is a section corresponding to a surjection $\mathcal{E} \rightarrow \mathcal{O}$. The locus of curves in $\mathcal{L}$ is a divisor, hence we have $a_{0}=a_{1}=\cdots=a_{n-1}=0$. Finally, from $-K_{X} \cdot \mathcal{L}=1$ we get $a_{n}=1$.

Assume now that $[C]$ is not extremal in $\mathrm{NE}(X)$. Since for a general $x \in X$ we have $\operatorname{dim} \operatorname{Locus}\left(C_{x}\right)=n-1$ by Proposition 2.5, in view of Theorem 2 of [5] this implies that $C$ is not a quasi-unsplit family.

Let $\ell^{1}+\ell^{2}$ be a reducible cycle in $\mathcal{C}$ whose components are not numerically proportional to $C$. For a general $x \in X$ we have seen that $\operatorname{Locus}\left(C_{x}\right)$ is a divisor $D_{x} ; D_{x}$ cannot contain curves numerically proportional to $\ell^{i}$, hence, if $D_{x} \cdot \ell^{i} \neq 0$ then, for every point $y$ in the locus of the corresponding family $\mathcal{L}^{i}$, we have $\operatorname{dim} \operatorname{Locus}\left(\mathcal{L}_{y}^{i}\right)=1$.

Assume, up to exchanging indexes, that $D_{x} \cdot \ell^{1} \neq 0$. Then, since $\mathcal{L}^{1}$ is not a covering family, by Proposition 2.5 we have $-K_{X} \cdot \mathcal{L}^{1}=1$, and thus $-K_{X} \cdot \mathcal{L}^{2}=n-1$.

By Lemma 2.7, $\mathrm{N}_{1}\left(D_{x}, X\right)=\langle[C]\rangle$; in particular, since $C$ is a dominating family, $D_{x \mid D_{x}}$ is nef. Since $D_{x}$ is effective, it follows that it is nef.

The nef divisor $D_{x}$ is trivial on $E_{2}=\operatorname{Locus}\left(\mathcal{L}_{x}^{2}\right)$, hence $\left[\mathcal{L}^{2}\right]$ generates an extremal ray, which is birational and of length $n-1$, so it corresponds to the blow-up of a smooth point in a smooth $X^{\prime}$.

Let $W$ be a minimal dominating family of rational curves for $X^{\prime}$, and let $W^{*}$ be the family of deformations of the strict transform of a curve in $W$. We have 
that $\left[W^{*}\right]=[C]$, since $E_{2}$ is trivial on both and $X$ does not carry a covering family of lines. Therefore

$$
-K_{X^{\prime}} \cdot W=-K_{X} \cdot W^{*}=-K_{X} \cdot C=n
$$

and $X^{\prime}$ is a smooth quadric by Theorem 0.1 (3) in [14]; in particular $X$ has another contraction whose exceptional locus is $E$, which is the blow-up of $\mathbb{P}^{n}$ along a smooth quadric of codimension 2.

Now we will show that, up to numerical equivalence, $\mathcal{B}^{\prime}$ contains only one pair.

Proposition 5.2. Let $(X, H)$ be an $R C C$-manifold with respect to $V$, not covered by lines, and of Picard number two. Then, up to numerical equivalence, $\mathcal{B}^{\prime}$ contains only one pair $(\mathcal{L}, C)$.

Proof. We will prove the proposition by contradiction.

By Proposition 5.1, we can assume that there are no divisors of $V$-lines of the second kind. We choose $\left(\mathcal{L}^{1}, C^{1}\right)$ to be a pair such that $m:=-K_{X} \cdot \mathcal{L}^{1}$ is maximum among the anticanonical degrees of families belonging to pairs in $\mathcal{B}^{\prime}$; since there are no divisors of the second kind we have $m>1$.

Since we are assuming that $\mathcal{B}^{\prime}$ contains a pair $\left(\mathcal{L}^{2}, C^{2}\right)$ with $\left[\mathcal{L}^{2}\right] \neq\left[\mathcal{L}^{1}\right]$ we have that $m \geq n / 2$ by formula (4.2).

Step 1. $E_{1} \cdot C^{2}=0$.

From the maximality of $m$ it follows that $-K_{X} \cdot \mathcal{L}^{2}<-K_{X} \cdot \mathcal{L}^{1}$, hence that $-K_{X} \cdot C^{2}>-K_{X} \cdot C^{1}$. Notice that the numerical class of $C^{2}$ cannot be proportional to $\left[\mathcal{L}^{1}\right]$, otherwise $-K_{X} \cdot C^{2}=2 m \geq n$, and the divisor of $V$-lines associated with the pair $\left(\mathcal{L}^{2}, C^{2}\right)$ would be of the second kind.

Therefore, if $E_{1} \cdot C^{2}>0$, then for a general $x \in X$ we have, by Lemma 2.7 and by Proposition 2.5, that

$$
\operatorname{dim} \operatorname{Locus}\left(\mathcal{L}^{1}\right)_{\operatorname{Locus}\left(C^{2}\right)_{x}} \geq-K_{X} \cdot C^{2}-K_{X} \cdot \mathcal{L}^{1}-2 \geq n,
$$

a contradiction, since $\mathcal{L}^{1}$ is not a covering family.

Step 2. The adjoint divisor $D:=K_{X}+m H$ is nef.

If this is not the case, since $D \cdot \mathcal{L}^{1}=0$ and $D \cdot V>0$, there is an extremal ray $R$ on the side of $\left[\mathcal{L}^{1}\right]$ with respect to $[V]$ on which $D$ is negative. Denote by $\varphi$ the associated contraction and let $W$ be a family of rational curves such that $\overline{\operatorname{Locus}(W)}=\operatorname{Exc}(\varphi)$ whose degree with respect to $H$ is minimal.

Every fiber of the contraction $\varphi$ has dimension greater than $m$. If $\varphi$ is birational then this follows from Proposition 2.10, since $l(R)>m$. If else $\varphi$ is of fiber type then a general fiber $F$ contains $\operatorname{Locus}(W)_{x}$ for some $x$, hence we have

$$
\operatorname{dim} F \geq \operatorname{dim} \operatorname{Locus}(W)_{x} \geq m H \cdot W-1>m,
$$

where the last inequality follows from the fact that $X$ is not covered by lines and that $m>1$. 
It follows that $E_{1} \cap \operatorname{Exc}(\varphi)=\emptyset$. In fact, if this were not the case, then $E_{1}$ would meet a fiber of $\varphi$, and in this case their intersection would contain a curve, contradicting the fact that $R \notin \mathrm{NE}\left(E_{1}, X\right)$ (cf. Lemma 4.1).

Therefore $E_{1} \cdot R=0$. Hence, by Step $1,\left[C^{2}\right] \in R$. Since $C^{2}$ is a dominating family, $\varphi$ is a fiber type contraction, contradicting $E_{1} \cap \operatorname{Exc}(\varphi)=\emptyset$.

Step 3. The contraction associated with a multiple of $D$ is a smooth blow-up.

Let $\varphi: X \rightarrow X^{\prime}$ be the contraction associated with $R:=\mathbb{R}_{+}\left[\mathcal{L}^{1}\right]$; let $W$ be a family of rational curves such that $\overline{\operatorname{Locus}(W)}=\operatorname{Exc}(\varphi)$ whose degree with respect to $H$ is minimal.

If $\varphi$ is of fiber type, then $H \cdot W \geq 2$, since $X$ is not covered by lines. Therefore, a general fiber of $\varphi$ has dimension $\geq 2 m-1$. Let $x$ be a general point. Then

$$
\operatorname{dim} F+\operatorname{dim} \operatorname{Locus}\left(C^{1}\right)_{x} \geq 2 m-1+(n-m)=n+m-1>n,
$$

a contradiction.

Therefore $\varphi$ is birational. In particular there exists an irreducible divisor which is negative on $R$ and therefore contains $\operatorname{Exc}(\varphi)$. So this divisor is $E_{1}$ and $E_{1} \cdot R<0$. Recall that, by construction, we have $E_{1}=\operatorname{Locus}\left(\mathcal{L}^{1}\right)_{\operatorname{Locus}\left(C^{1}\right)_{x}}$ for a general $x \in X$; it follows that any fiber $F$ of $\varphi$ meets Locus $\left(C^{1}\right)_{x}$, hence, by Proposition 2.5, we have

$$
n \geq \operatorname{dim} F+\operatorname{dim} \operatorname{Locus}\left(C^{1}\right)_{x} \geq-K_{X} \cdot \mathcal{L}^{1}-K_{X} \cdot C^{1}-1=n,
$$

hence equality holds. In particular for any fiber of $\varphi$ we have $\operatorname{dim} F=-K_{X} \cdot \mathcal{L}^{1}$, thus $\varphi$ is a smooth blow-up by Theorem 2.11 .

Step 4. Conclusion.

By Step $1, E_{1} \cdot C^{2}=0$, hence $\left[C^{2}\right]$ is in the interior of the cone $\left\langle\left[\mathcal{L}^{1}\right],[V]\right\rangle$, and so $E_{1} \cdot V>0$, which in turn implies $E_{1} \cdot C^{1} \geq 2$. By formula (4.1) we have

$$
-K_{X} \cdot C^{2} \leq n+2-\left(-K_{X} \cdot C^{1}\right)=-K_{X} \cdot \mathcal{L}^{1}+1=m+1 .
$$

Let $\varphi: X \rightarrow X^{\prime}$ be the blow-up. The line bundle $H+E_{1}$ is trivial on $R$, hence there exists $H^{\prime} \in \operatorname{Pic}\left(X^{\prime}\right)$ such that $H+E_{1}=\varphi^{*} H^{\prime}$. Since $\rho_{X^{\prime}}=1$ we can write $-K_{X^{\prime}}=k H^{\prime}$. Since $X^{\prime}$ is Fano and $H+E_{1}$ is nef, we have $k>0$.

By the canonical bundle formula

$$
-K_{X}=-\varphi^{*} K_{X^{\prime}}-m E_{1}=k H+(k-m) E_{1}
$$

we have

$$
m+1 \geq-K_{X} \cdot C^{2}=-\varphi^{*} K_{X^{\prime}} \cdot C^{2}=2 k
$$

and

$$
n+1-m=-K_{X} \cdot C^{1}=2 k+(k-m)\left(E_{1} \cdot C^{1}\right) .
$$

Recalling that $E_{1} \cdot C^{1} \geq 2$ we have

$$
n+1-m \leq 2 k-2(m-k) \leq 2,
$$

where the last inequality follows from (5.3). 
Since $m \leq n-1$, the only possibility is that all the inequalities are equalities; in particular $m=n-1$ and $E_{2}$ is of the second kind, a contradiction.

Now we will prove the first part of Theorem 1.1 under the assumption that the Picard number of $X$ is two.

Theorem 5.3. Let $(X, H)$ be an $R C C$-manifold with respect to $V$, not covered by lines, and of Picard number two. Then there exists a polarized manifold $\left(X^{\prime}, H^{\prime}\right)$ of Picard number one not covered by lines and an elementary contraction $\varphi_{\sigma}: X \rightarrow X^{\prime}$ expressing $X$ as a blow-up of $X^{\prime}$ along a smooth center $T$, with exceptional divisor $E$, such that $H \simeq \varphi_{\sigma}^{*} H^{\prime}-E$. The pair $\left(X^{\prime}, H^{\prime}\right)$ is $R C C$ with respect to $V^{\prime}$, the family of deformations of the image of a general curve parametrized by $V$.

Proof. By Proposition 5.2, we know that, up to numerical equivalence, there is only one pair $(\mathcal{L}, C)$ in $\mathcal{B}^{\prime}$. Let $E$ be the corresponding divisor of $V$-lines. We claim that $E \cdot V=0$.

Assume by contradiction that $E \cdot V>0$. Let $x \in X$ be a general point, and consider the following diagram:

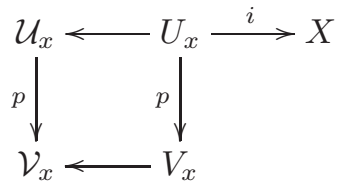

By our assumptions, the inverse image $i^{-1}(E)$ dominates $V_{x}$; moreover, since $V$ is generically unsplit $i_{\mid i^{-1}(E)}: i^{-1}(E) \rightarrow E$ is a generically finite map, hence it is dominating, since $\operatorname{dim} V_{x}=\operatorname{dim} i^{-1}(E)=\operatorname{dim} E$.

Let $y \in E$ be a general point and let $F$ be a component of $\operatorname{Locus}(\mathcal{L})_{y}$. We can find a (non-complete) curve $\Gamma^{0}$ in $i^{-1}(F)$. Let $B^{0}$ be $p\left(\Gamma^{0}\right)$ and $S^{0}:=p^{-1}\left(B^{0}\right)$; notice that every curve parametrized by $B^{0}$ meets $\Gamma^{0}$.

Let $\bar{B}$ be the closure of $B^{0}$ in $\mathcal{V}_{x}$, let $\bar{S}$ be $p^{-1}(\bar{B})$, let $\nu: B \rightarrow \bar{B}$ be the normalization, $S=B \times{ }_{B} \bar{S}$ and $\Gamma$ the curve in $S$ whose image in $S^{0}$ is $\Gamma^{0}$. Notice that, by construction, the image in $X$ of $\Gamma$ is a curve contained in $F$, hence it is numerically proportional to $[\mathcal{L}]$.

By Proposition II.4.19 in [13] every curve in $S$ is algebraically equivalent to a linear combination with rational coefficients of a section $C_{0}$ such that $i\left(C_{0}\right)=x$, and of the irreducible components of fibers of $p_{\mid S}$ (in the quoted proposition, take $X=S, T=B$ and $\left.Z=C_{0}\right)$.

The images of irreducible components of fibers of $p$ are irreducible curves whose numerical class is $[V],[\mathcal{L}]$ or $[C]$. If all the fibers of $p: S \rightarrow B$ are irreducible, then in $S$ we can write

$$
\Gamma \equiv \alpha C_{0}+\beta f
$$

with $[i(f)]=[V]$, and we get that $i(\Gamma)$ is numerically proportional to $V$, a contradiction. So we can write

$$
\Gamma \equiv \alpha C_{0}+\sum \gamma_{i} C_{i}+\sum \delta_{j} \ell_{j}
$$


with $\left[i\left(C_{i}\right)\right]=[C],\left[i\left(\ell_{j}\right)\right]=[\mathcal{L}]$. Hence,

$$
[i(\Gamma)]=\sum \gamma_{i}[C]+\sum \delta_{j}[\mathcal{L}]
$$

and we get $\sum \gamma_{i}=0$.

Since $i(\Gamma) \not \supset x$, then $\Gamma \cdot C_{0}=0$. Recalling that $x$ is not contained in any line, we get

$$
\alpha C_{0}^{2}+\sum \gamma_{i}=0
$$

since $C_{0}$ goes to a point then $C_{0}^{2}<0$, hence $\alpha=0$. This implies that, for a general fiber $f$ we have $f \cdot \Gamma=0$, a contradiction.

The statement now follows applying Proposition 4.3.

\section{Proof of Theorem 1.1}

Proof. Let $\mathcal{B}^{\prime}$ be as in Section 4 and let $\mathcal{B}=\left\{\left(\mathcal{L}^{i}, C^{i}\right)\right\}_{i=1}^{k}$ be a maximal set of pairs in $\mathcal{B}^{\prime}$ such that $[V],\left[\mathcal{L}^{1}\right], \ldots,\left[\mathcal{L}^{k}\right]$ are numerically independent, and chosen in the following way: if there exists a pair in $\mathcal{B}^{\prime}$ whose divisor of $V$-lines is of the second kind we choose it to be $\left(\mathcal{L}^{1}, C^{1}\right)$; if no such pair exists, but there is a pair whose divisor of $V$-lines is of the third kind we choose it to be $\left(\mathcal{L}^{1}, C^{1}\right)$.

Since we have already proved the first part of the Theorem for $\rho_{X}=2$ we can assume that $k \geq 2$.

We start by showing that all the divisors of the first kind in $\mathcal{B}$ correspond to blow-ups at points, and can be simultaneously contracted; by Proposition 4.3 this will be the case if $E_{i} \cdot V=0$ for every such divisor.

If $E_{1}$ is not of the first kind, then $E_{i} \cdot C^{1}=0$ for every divisor $E_{i}$ of the first kind. We already know from Lemma 4.2 that $E_{i} \cdot \mathcal{L}^{1}=0$ for every divisor of $V$-lines in $\mathcal{B}$ with $i \neq 1$, hence we get that $E_{i} \cdot V=0$ for every divisor of the first kind. So we are left with the case in which every divisor of $V$-lines in $\mathcal{B}$ (and in $\mathcal{B}^{\prime}$, by our choice of $\mathcal{B}$ ) is of the first kind. Again we want to prove that $E_{i} \cdot V=0$ for every $i$.

To this aim we will consider the reduction morphism associated with the adjoint divisor $D:=K_{X}+(n-1) H$, which we claim is nef and big.

Assume first that $D$ is not nef. Then there exists an extremal ray $R$ of $X$ which has length $l(R) \geq n$. The associated contraction $\varphi_{R}$ is of fiber type and has fibers of dimension $\geq n-1$ by Proposition 2.10. Let $E$ be a first kind divisor and pick $x \in E$; let $F_{x}$ be the fiber of $\varphi_{R}$ passing through $x$. Then

$$
\operatorname{dim}\left(E \cap F_{x}\right) \geq \operatorname{dim} E+\operatorname{dim} F_{x}-n>0
$$

a contradiction, since $\left[\mathcal{L}^{1}\right] \notin R$, being $D \cdot \mathcal{L}^{1}=0$ and $D \cdot R<0$.

Therefore $D$ is nef and defines an extremal face $\tau \subset \mathrm{NE}(X)$, with associated contraction $\varphi_{\tau}: X \rightarrow Y$.

Assume now by contradiction that $D$ is not big, i.e., that $\varphi_{\tau}$ is of fiber type, and let $W$ be a minimal dominating family of rational curves such that $[W] \in \tau$. 
Then $-K_{X} \cdot W=(n-1) H \cdot W \leq n$, where the last inequality follows from the fact that $W$ is locally unsplit and $\rho_{X} \geq 1$. Therefore $H \cdot W=1$, contradicting our assumptions that $X$ is not covered by lines.

Therefore $D$ is nef and big and we can apply Theorem 7.3.2 in [3] to get that $Y$ is smooth and $\varphi_{\tau}$ is the blow-up of $Y$ along $t$ distinct points. Since $D \cdot \mathcal{L}^{i}=0$ for every $i$ we have $t \geq k$. On the other hand, since $\rho_{X}=k+1$ we have $t \leq k$, so $\varphi_{\tau}$ is a blow-up of a smooth $X^{\prime}$ along $k$ points, and the exceptional divisors are the divisors of $V$-lines.

Take a curve $B$ in $X^{\prime}$ not containing the centers of the blow-up and not meeting the images of the conics parameterized by the families $C^{j}$ belonging to pairs in $\mathcal{B}^{\prime}$ passing through a fixed general point $x$. Since all the divisors of $V$-lines are of the first kind there is a finite number of these conics through $x$.

By construction the strict transform $\widetilde{B}$ does not meet cycles in $\mathcal{V}_{x}$ whose components are not proportional to $V$, hence its numerical class is proportional to $V$. Since $\widetilde{B}$ does not meet the $E_{i}$ 's we have $E_{i} \cdot \widetilde{B}=0$ for every $i$, hence $E_{i} \cdot V=0$.

We can thus apply Proposition 4.3 to the set of the divisors of the the first kind to get a new pair $\left(X^{\prime \prime}, H^{\prime \prime}\right)$. If $\rho_{X^{\prime \prime}} \leq 2$ then we are done, otherwise every divisor of $V^{\prime \prime}$-lines is of the third kind.

By Lemma 4.2 these divisors are disjoint and have intersection number zero with $V$, so we can apply again Proposition 4.3. In any case we finally get to a pair $\left(X^{\prime}, H^{\prime}\right)$ with $\rho_{X^{\prime}}=1$ and to a family $V^{\prime}$ as in the statement.

We now come to the description of $\left(X^{\prime}, H^{\prime}\right)$.

If $V^{\prime}$ is a minimal dominating family then $X^{\prime} \simeq \mathbb{P}^{n}$ by Theorem 1.1 of [11]; if this is not the case, then there is a dominating family $W$ of rational curves in $X^{\prime}$ such that $-K_{X^{\prime}} \cdot W<n+1$.

Let $W^{*}$ be the family of deformations in $X$ of the strict transform of a curve in $W$. Since $W$ is dominating, a general curve parametrized by $W$ does not meet the union $T=\cup T_{i}$ of the centers of the blow-up. Hence $E_{i} \cdot W^{*}=0$ for every $i$ and $W^{*}$ is numerically proportional to $V$.

Since $X$ is not covered by lines we can assume that $H \cdot W^{*}=2$. Using again the canonical bundle formula and the projection formula we get

$$
-K_{X^{\prime}} \cdot W=-K_{X} \cdot W^{*}=-\frac{2}{3} K_{X} \cdot V=\frac{2}{3}(n+1) .
$$

We know that $H=\varphi^{*} H^{\prime}-\sum E_{i}$, hence $H^{\prime} \cdot W=2$ and $\varphi^{*} H^{\prime} \cdot C^{i}=3$. Therefore, $H^{\prime}$ is the fundamental divisor of $X^{\prime}$, and the index of $X^{\prime}$ is $\frac{n+1}{3}$.

The family $W^{*}$ is locally unsplit since $X$ is not covered by lines. For a general $x$ we have

$$
\operatorname{dim} \operatorname{Locus}\left(W^{*}\right)_{x} \geq-K_{X} \cdot W^{*}-1 \geq \frac{2 n-1}{3} .
$$

Notice that $\operatorname{codim}\left(T_{i}\right)-1=-K_{X} \cdot \mathcal{L}^{i}$, hence

$$
\operatorname{dim} \operatorname{Locus}\left(C^{i}\right)_{x}=-K_{X} \cdot C_{i}-1=n+K_{X} \cdot \mathcal{L}^{i}=\operatorname{dim} T_{i}+1 .
$$

If for some $i$ we have $\operatorname{dim} T_{i} \geq(n+1) / 3$ then we get a contradiction considering the intersection $\operatorname{Locus}\left(C^{i}\right)_{x} \cap \operatorname{Locus}\left(W^{*}\right)_{x}$ for any $i$. 


\section{RCC Fano manifolds}

In this section we will show how restricting to RCC Fano manifolds leads to stronger results.

Proposition 7.1. With the assumptions of Theorem 1.1, if $X$ is a Fano manifold and $\left(X^{\prime}, H^{\prime}\right) \nsucceq\left(\mathbb{P}^{n}, \mathcal{O}(3)\right)$, then $\operatorname{dim} T_{i}=\frac{n-2}{3}$ for every $i$.

Proof. We keep the notation of the proof of Theorem 1.1. The $\operatorname{rc}\left(\mathcal{W}^{*}\right)$-fibration contracts curves parametrized by $V$, hence it goes to a point. Therefore $W^{*}$ cannot be a quasi-unsplit family, otherwise $\rho_{X}=1$. Hence, there is a reducible cycle $l^{*}+\bar{l}^{*}$ in $\mathcal{W}^{*}$ whose components are not numerically proportional.

For at least one $i$ the divisor $E_{i}$ is not trivial on both $l^{*}$ and $\bar{l}^{*}$, hence, up to exchanging them, we can assume $E_{i} \cdot l^{*}<0$; therefore $\left[l^{*}\right] \in R^{i}$ and $-K_{X} \cdot l^{*}=$ $-K_{X} \cdot \mathcal{L}^{i}$, so

$$
-K_{X} \cdot \mathcal{L}^{i}<-K_{X} \cdot W^{*}=\frac{2(n+1)}{3}
$$

and we get $\operatorname{codim} T_{i} \leq 2 / 3(n+1)$, which, combined with the bound obtained in Theorem 1.1 gives $\operatorname{dim} T_{i}=\frac{n-2}{3}$.

\subsection{Higher Picard number}

In this section we are going to prove that a Fano RCC manifold has Picard number at most three, and to classify those of Picard number three.

We will need the following result from basic projective geometry; the proof we give here was pointed out to us by Francesco Russo.

Lemma 7.2. Let $T \subset \mathbb{P}^{n}$ be a projective manifold, and denote by $\mathcal{S}(T)$ its secant variety. Then,

1) if $\operatorname{dim} \mathcal{S}(T)=\operatorname{dim} T$ then $T$ is a linear subspace of $\mathbb{P}^{n}$;

2) if $\operatorname{dim} \mathcal{S}(T)=\operatorname{dim} T+1$ then $T$ is a hypersurface of $\langle T\rangle \simeq \mathbb{P}^{\operatorname{dim} T+1}$.

Proof. We denote by $\mathbb{T}_{z} Z$ the projective tangent space to a variety $Z$ at a point $z$. If $t \in T$, we denote by $\mathcal{S}(t, T)$ the relative secant variety of $T$ with respect to $t$.

First of all notice that, for every $t \in T$,

$$
T \subseteq \mathcal{S}(t, T) \subseteq \mathbb{T}_{t} \mathcal{S}(t, T) \subseteq \mathbb{T}_{t} \mathcal{S}(T)
$$

Assume that $\operatorname{dim} \mathcal{S}(T)=\operatorname{dim} T$. Clearly $\mathcal{S}(T)=T$. Let $t \in T$ be a point of $T$. By (7.1) and by our assumptions we have that

$$
T \subseteq \mathbb{T}_{t} \mathcal{S}(T)=\mathbb{T}_{t} T=\mathbb{P}^{\operatorname{dim} T}
$$

and hence $T=\mathbb{P}^{\operatorname{dim} T}$ since $T$ and $\mathbb{T}_{t} T$ are irreducible varieties of the same dimension $\operatorname{dim} T$.

Now we suppose that $\operatorname{dim} \mathcal{S}(T)=\operatorname{dim} T+1$. For a general point $t \in T$,

$$
T \subsetneq \mathcal{S}(t, T) \subseteq \mathcal{S}(T)
$$


and hence

$$
\operatorname{dim} T<\operatorname{dim} \mathcal{S}(t, T) \leq \operatorname{dim} \mathcal{S}(T)=\operatorname{dim} T+1 .
$$

This implies that for a general point $t \in T$ we have $\mathcal{S}(t, T)=\mathcal{S}(T)$, hence for a general point $x \in \mathcal{S}(T) \backslash T$ there exists $t^{\prime} \in T$ such that

$$
x \in\left\langle t, t^{\prime}\right\rangle \subset \mathcal{S}(t, T)=\mathcal{S}(T) .
$$

From this it follows that a general point $t \in T$ is contained in $\mathbb{T}_{x} \mathcal{S}(T)$ and that

$$
\mathcal{S}(T) \subseteq\langle T\rangle \subseteq \mathbb{T}_{x} \mathcal{S}(T),
$$

where $\langle T\rangle$ is the linear span of $T$ in $\mathbb{P}^{n}$.

Now, by the generality of $x \in \mathcal{S}(T)$ we know that $\operatorname{dim} \mathbb{T}_{x} \mathcal{S}(T)=\operatorname{dim} \mathcal{S}(T)$ and hence

$$
\mathcal{S}(T)=\mathbb{T}_{x} \mathcal{S}(T)=\mathbb{P}^{\operatorname{dim} \mathcal{S}(T)}=\mathbb{P}^{\operatorname{dim} T+1},
$$

which concludes the proof.

Proof of Theorem 1.2. By Theorem 1.1 and Proposition 7.1, we know that $(X, H)$ is the blow-up of $\left(X^{\prime}, H^{\prime}\right)$ along disjoint centers. Assume that $\rho_{X}>3$ and let $\left(\mathcal{L}^{i}, C^{i}\right),\left(\mathcal{L}^{k}, C^{k}\right)$ be two independent pairs in $\mathcal{B}^{\prime}$ with associated divisors of $V$ lines $E_{i}$ and $E_{k}$.

Since $E_{i}$ cannot contain curves of $C^{k}$, but $C^{k}$ is dominating, it follows that there exists a reducible cycle $l_{k}+\bar{l}_{k}$ in $\mathcal{C}^{k}$ such that $E_{i} \cdot l_{k}<0$. This implies that $\left[l_{k}\right] \in \mathrm{NE}\left(E_{i}, X\right) \subset \Pi_{i}$. Notice that $H+E_{i}$ is nef on $\Pi_{i}$, hence $E_{i} \cdot l_{k}=-1$. Since both $H$ and $E_{i}$ have the same intersection number with $l_{k}$ and $\mathcal{L}^{i}$, then $\left[l_{k}\right]=\left[\mathcal{L}^{i}\right]$.

Recalling that $X$ is Fano, this implies that $-K_{X} \cdot C^{k} \geq-K_{X} \cdot \mathcal{L}^{i}+1$, and so we get that $-K_{X} \cdot\left(\mathcal{L}^{k}+\mathcal{L}^{i}\right) \leq n$. Hence, by formula (4.2) that

$$
-K_{X} \cdot\left(\mathcal{L}^{k}+\mathcal{L}^{i}\right)=n .
$$

Notice that $-K_{X} \cdot \mathcal{L}^{i}$ is the length of the contraction of the extremal ray generated by $\left[\mathcal{L}^{i}\right]$. In particular, if $T_{i}$ is the image of $E_{i}$ via $\pi$, we have $\operatorname{dim} T_{i}=n-\left(-K_{X}\right.$. $\left.\mathcal{L}^{i}\right)-1$, hence, for $i \neq k$

$$
\operatorname{dim} T_{k}+\operatorname{dim} T_{i}=n-2 .
$$

By Proposition 7.1 it follows that $\left(X^{\prime}, H^{\prime}\right) \simeq\left(\mathbb{P}^{n}, \mathcal{O}(3)\right)$.

If $\rho_{X}>3$, combining with formula (4.1) we have that, for every $i$,

$$
-K_{X} \cdot C^{i}=\frac{n+2}{2} \text { and } \quad-K_{X} \cdot \mathcal{L}^{i}=\frac{n}{2}
$$

and hence $\operatorname{dim} T_{i}=\frac{n-2}{2}$ for every $i$.

Recall now that $H=\pi^{*} \mathcal{O}(3)-\sum E_{i}$ is ample. Take two of the centers $T_{1}$ and $T_{2}$ and consider their join. By Proposition 11.37 in [8], it has dimension $n-1$, hence it meets some other center $T_{3}$. Take a line $\ell$ meeting three centers. Then $\left(\pi^{*} \mathcal{O}(3)-\sum E_{i}\right) \cdot \ell \leq 0$, a contradiction. Therefore $\rho_{X} \leq 3$. 
Assume now that $\rho_{X}=3$. Let $\mathcal{S}\left(T_{1}\right)$ be the secant variety of $T_{1}$. Suppose that $\operatorname{dim} \mathcal{S}\left(T_{1}\right) \geq \operatorname{dim} T_{1}+2$. Then

$$
\operatorname{dim}\left(\mathcal{S}\left(T_{1}\right) \cap T_{2}\right) \geq \operatorname{dim} \mathcal{S}\left(T_{1}\right)+\operatorname{dim} T_{2}-n \geq \operatorname{dim} T_{1}+2+\operatorname{dim} T_{2}-n=0,
$$

i.e., there is a line $l$ in $\mathbb{P}^{n}$ which meets $T_{1}$ in two points and $T_{2}$ in a point; as above we show that $H \cdot l \leq 0$.

It follows by Lemma 7.2 that either $T_{i}$ is a linear space or a hypersurface in a linear space of dimension $\operatorname{dim} T_{i}+1$.

Notice also that from the ampleness of $H$ it follows that there cannot exist trisecant lines of $T_{i}$ in $\mathbb{P}^{n}$, and hence, if $T_{i}$ is not a linear space, then it is a hyperquadric, and we can prove that $\operatorname{dim} T_{i} \geq \frac{n}{2}-1$.

In fact, considering the strict transform $l$ of a secant line of $T_{i}$ and recalling that $X$ is Fano, by the canonical bundle formula, we get

$$
1 \leq-K_{X} \cdot l=(n+1)-2 \operatorname{codim}\left(T_{i}\right)+2 \quad \Longrightarrow \quad \operatorname{dim} T_{i} \geq \frac{n}{2}-1 .
$$

Therefore:

1. If $\operatorname{dim} \mathcal{S}\left(T_{1}\right)=\operatorname{dim} T_{1}$ and $\operatorname{dim} \mathcal{S}\left(T_{2}\right)=\operatorname{dim} T_{2}$, then $X$ is the blow-up of $\mathbb{P}^{n}$ along two disjoint linear subspaces.

2. If $\operatorname{dim} \mathcal{S}\left(T_{1}\right)=\operatorname{dim} T_{1}$ and $\operatorname{dim} \mathcal{S}\left(T_{2}\right)=\operatorname{dim} T_{2}+1$, then $X$ is the blow-up of $\mathbb{P}^{n}$ along a linear subspace $T_{1}$ and along a quadric $T_{2} \subset \Lambda_{2} \simeq \mathbb{P}^{\text {dim } T_{2}+1}$ such that $\operatorname{dim} T_{1} \leq \frac{n}{2}-1$. Moreover $\Lambda_{2}$ and $T_{1}$ must be disjoint, because there cannot exist lines in $\mathbb{P}^{n}$ which meet $T_{1}$ in a point and $T_{2}$ in two points.

3. If $\operatorname{dim} \mathcal{S}\left(T_{1}\right)=\operatorname{dim} T_{1}+1$ and $\operatorname{dim} \mathcal{S}\left(T_{2}\right)=\operatorname{dim} T_{2}+1$, then $X$ is the blow-up of $\mathbb{P}^{n}$ along two quadrics $T_{1}, T_{2}$ such that $\operatorname{dim} T_{1}=\operatorname{dim} T_{2}=\frac{n}{2}-1$ (clearly $n$ is even). Notice also that $T_{i} \subset \Lambda_{i} \simeq \mathbb{P}^{\frac{n}{2}}$, and $\operatorname{dim}\left(\Lambda_{1} \cap \Lambda_{2}\right)=0$ because there cannot exist trisecant lines of $T_{1} \cup T_{2}$.

\subsection{Picard number two: some examples}

Let $(X, H)$ be an RCC Fano manifold of Picard number two obtained as the blowup of $\mathbb{P}^{n}$ along a smooth center $T$. Denote by $\varphi: X \rightarrow \mathbb{P}^{n}$ the blow-up contraction and by $E$ the exceptional divisor. Then, $H=\varphi^{*} \mathcal{O}_{\mathbb{P}^{n}}(3)-E$.

By the ampleness of the anticanonical bundle

$$
-K_{X}=\varphi^{*} \mathcal{O}_{\mathbb{P}^{n}}(n+1)-(\operatorname{codim} T-1) E,
$$

we have that, if $T$ is not a linear space, then $\operatorname{dim} T>(n-3) / 2$. To see this, just compute the intersection of $-K_{X}$ with the strict transform of a secant line of $T$. Moreover, by the ampleness of $H$ we get that $T$ has no trisecants. A large class of examples is given by the following.

Proposition 7.3. Let $T \subset \mathbb{P}^{n}$ be a smooth subvariety of dimension $t>(n-3) / 2$ which is scheme theoretically defined by quadratic equations. Then the pair $(X, H)=$ $\left(B l_{T}\left(\mathbb{P}^{n}\right), 3 \mathcal{H}-E\right)$ is a Fano $R C C$ manifold. Moreover $X$ is covered by $H$-lines if and only if $\mathcal{S}(T)=\mathbb{P}^{n}$. 
Proof. Consider the rational map $\psi: \mathbb{P}^{n}--\rightarrow \mathbb{P}^{N}$ given by the system of quadrics through $T$ and the resolution of this map:

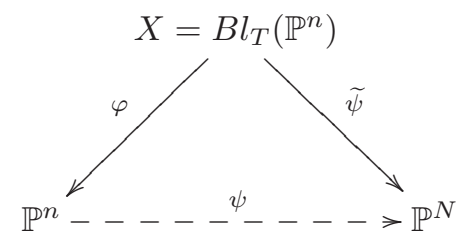

The morphism $\widetilde{\psi}$ is given by the linear system $\left|\varphi^{*} \mathcal{O}_{\mathbb{P}^{n}}(2)-E\right|$. Hence, it contracts the strict transforms of the (bi)secants to $T$. Using the canonical bundle formula we see that the intersection number of $-K_{X}$ with these curves is positive, and therefore $X$ is a Fano manifold.

The ampleness of $\varphi^{*} \mathcal{O}_{\mathbb{P}^{n}}(3)-E$ is now given by the Kleiman criterion. The family $V$ is the family of deformations of the strict transform of a general line in $\mathbb{P}^{n}$. The last statement follow from the fact that an $H$-line not contained in $E$ is the strict transform of a (bi)-secant line of $T$.

Remark 7.4. Some examples of manifolds obtained as in Proposition 7.3 can be found in [6], Cases (b1)-(b6) and (c1)-(c2).

\section{Examples}

Example 8.1. $(X, H) \simeq\left(B l_{\Lambda_{1}, \Lambda_{2}}\left(\mathbb{P}^{n}\right), 3 \mathcal{H}-E_{1}-E_{2}\right)$, where $B l_{\Lambda_{1}, \Lambda_{2}}\left(\mathbb{P}^{n}\right)$ is the blow-up of $\mathbb{P}^{n}$ along two disjoint linear spaces $\Lambda_{1} \simeq \mathbb{P}^{t}$ and $\Lambda_{2} \simeq \mathbb{P}^{n-2-t} ; E_{1}, E_{2}$ are the exceptional divisors of $\pi$ and $\mathcal{H}=\pi^{*} \mathcal{O}_{\mathbb{P}^{n}}(1)$. Denote by

- $R^{i}$ the extremal ray corresponding to the contraction of $E_{i}$;

- $\varepsilon_{i}$ the contraction associated with $R^{i}$;

- $\ell_{i}$ a minimal curve whose numerical class is in $R^{i}$;

- $\ell$ a curve which is the strict transform of a line meeting both $\Lambda_{1}$ and $\Lambda_{2}$ in a point;

- $D_{i}=\mathcal{H}-E_{i}$.

The line bundles $\mathcal{H}, D_{1}$ and $D_{2}$ are nef on $X$. The cone of curves is therefore contained in the intersection of the positive halfspaces of $\mathrm{N}_{1}(X)$ determined by them. By looking at the intersection numbers with the curves $\ell_{1}, \ell_{2}, \ell$,

\begin{tabular}{c|c|c|c} 
& $\ell_{1}$ & $\ell_{2}$ & $\ell$ \\
\hline $\mathcal{H}$ & 0 & 0 & 1 \\
\hline$D_{1}$ & 1 & 0 & 0 \\
\hline$D_{2}$ & 0 & 1 & 0 \\
\hline
\end{tabular}

we see that $\mathrm{NE}(X)$ is the intersection of those halfspaces, and that is spanned by three rays, $R^{1}=\mathbb{R}_{+}\left[\ell_{1}\right], R^{2}=\mathbb{R}_{+}\left[\ell_{2}\right], R^{3}=\mathbb{R}_{+}[\ell]$. Clearly the elementary contractions associated with $R^{1}$ and $R^{2}$ are the the blow-downs of $E_{1}$ and $E_{2}$. 
The elementary contraction associated with $R^{3}$ is divisorial, and its exceptional locus is the strict transform of the join $J\left(\Lambda_{1}, \Lambda_{2}\right)$. It is possible to show that this contraction is the blow-up of $\mathbb{P}^{n-t-1} \times \mathbb{P}^{t+1}$ along a smooth subvariety $\mathbb{P}^{n-t-2} \times \mathbb{P}^{t}$.

Description of the families of rational curves. In this example the family $V$ of cubics is the family of deformations of the strict transform of a general line of $\mathbb{P}^{n}$; the set $\mathcal{B}^{\prime}$ consists of two pairs, $\left(\mathcal{L}^{1}, C^{1}\right)$ and $\left(\mathcal{L}^{2}, C^{2}\right)$ : the families $\mathcal{L}^{i}$ are the families of lines contracted by the blow-down, while the families $C^{i}$ are the families of strict transforms of lines in $\mathbb{P}^{n}$ meeting one of the centers.

Curves in $C^{i}$ degenerate into a line contracted by $\varepsilon_{j}(i \neq j)$ and the strict transform of a line meeting both $\Lambda_{1}$ and $\Lambda_{2}$.

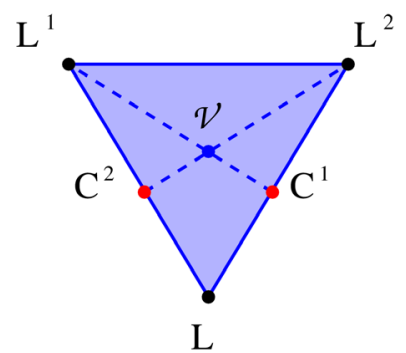

Example 8.2. $(X, H) \simeq\left(B l_{\Lambda_{1}, Q_{1}}\left(\mathbb{P}^{n}\right), 3 \mathcal{H}-E_{1}-E_{2}\right)$, where $B l_{\Lambda_{1}, Q_{1}}\left(\mathbb{P}^{n}\right)$ is the blow-up of $\mathbb{P}^{n}$ along a linear space $\Lambda_{1} \simeq \mathbb{P}^{t}$ and a smooth quadric $Q_{1} \subset \Lambda_{2} \simeq$ $\mathbb{P}^{n-1-t}$ such that $\Lambda_{1} \cap \Lambda_{2}=\emptyset ; E_{1}, E_{2}$ are the exceptional divisors of $\pi$ and $\mathcal{H}=$ $\pi^{*} \mathcal{O}_{\mathbb{P}^{n}}(1)$. Denote by

- $R^{i}$ the extremal ray corresponding to the contraction of $E_{i}$;

- $\varepsilon_{i}$ the contraction associated with $R^{i}$;

- $\ell_{i}$ a minimal curve whose numerical class is in $R^{i}$;

- $\bar{\ell}_{1}$ a curve which is the strict transform of a line meeting both $\Lambda_{1}$ and $Q_{1}$ in a point;

- $\bar{\ell}_{2}$ a curve which is the strict transform of a general line contained in $\Lambda_{2}$;

- $D_{1}=\mathcal{H}-E_{1}$;

- $D_{2}=2 \mathcal{H}-E_{2}$;

- $D_{3}=2 \mathcal{H}-E_{1}-E_{2}$.

The line bundles $\mathcal{H}, D_{1}$ and $D_{2}$ are nef on $X$. We want to show that also $D_{3}$ is nef.

Suppose by contradiction that there is an irreducible curve $C \subset X$ such that $D_{3} \cdot C<0$. Then $\left(\mathcal{H}-E_{1}\right) \cdot C<0$ or $\left(\mathcal{H}-E_{2}\right) \cdot C<0$.

Assume that $\left(\mathcal{H}-E_{2}\right) \cdot C<0$ (the other case is dealt with in a similar way). The map $\pi$ factors as $\varepsilon_{2} \circ \varepsilon_{1}$. Let $\widetilde{H}$ be a hyperplane of $\mathbb{P}^{n}$ which contains $\Lambda_{2}$ and let $H^{\prime}$ be the strict transform of $\widetilde{H}$ via $\varepsilon_{2}$. We have that

$$
\mathcal{H}-E_{2}=\varepsilon_{1}^{*} H^{\prime}
$$

and hence, by the projection formula, we get

$$
\left(\mathcal{H}-E_{2}\right) \cdot C=H^{\prime} \cdot \varepsilon_{1 *} C<0 .
$$


This implies that the curve $C$ is not contracted by $\varepsilon_{1}$ and that $\varepsilon_{1}(C)$ is contained in $H^{\prime}$. Since this holds for every hyperplane containing $\Lambda_{2}$ we have $\pi(C) \subset \Lambda_{2}$, so either $C$ is contained in the strict transform of $\Lambda_{2}$ or $C \subset E_{2}$.

Since

$$
E_{2}=\mathbb{P}\left(\mathcal{N}_{Q_{1} / \mathbb{P}^{n}}^{*}\right) \simeq \mathbb{P}\left(\mathcal{O}(-2) \oplus \mathcal{O}(-1)^{\oplus n-2-t}\right),
$$

with the strict transform of $\Lambda_{2}$ cutting the section corresponding to the surjection $\mathcal{N}_{Q_{1} / \mathbb{P}^{n}}^{*} \rightarrow \mathcal{O}(-2)$ we have that $\mathrm{NE}\left(E_{2}, X\right)=\left\langle\left[\bar{\ell}_{2}\right],\left[\ell_{2}\right]\right\rangle$, while every curve contained in the strict transform of $\Lambda_{2}$ is numerically proportional to $\left[\bar{\ell}_{2}\right]$.

Since $D_{3} \cdot \ell_{2}=1$ and $D_{3} \cdot \bar{\ell}_{2}=0$, we get a contradiction which proves the nefness of $D_{3}$.

We have four nef line bundles: $\mathcal{H}, D_{1}, D_{2}$ and $D_{3}$. The cone of curves is therefore contained in the intersection of the positive halfspaces of $\mathrm{N}_{1}(X)$ determined by them. By looking at the intersection numbers with the four curves $\ell_{1}, \ell_{2}, \bar{\ell}_{1}, \bar{\ell}_{2}$,

\begin{tabular}{c|c|c|c|c} 
& $\ell_{1}$ & $\ell_{2}$ & $\bar{\ell}_{1}$ & $\bar{\ell}_{2}$ \\
\hline $\mathcal{H}$ & 0 & 0 & 1 & 1 \\
\hline$D_{1}$ & 1 & 0 & 0 & 1 \\
\hline$D_{2}$ & 0 & 1 & 1 & 0 \\
\hline$D_{3}$ & 1 & 1 & 0 & 0 \\
\hline
\end{tabular}

we see that $\mathrm{NE}(X)$ is the intersection of these halfspaces, that it is spanned by four rays, $R^{1}=\mathbb{R}_{+}\left[\ell_{1}\right], R^{2}=\mathbb{R}_{+}\left[\ell_{2}\right], R^{3}=\mathbb{R}_{+}\left[\bar{\ell}_{1}\right], R^{4}=\mathbb{R}_{+}\left[\bar{\ell}_{2}\right]$ and that the position of these rays is as in the next figure, which shows a cross section of $\mathrm{NE}(X)$ :

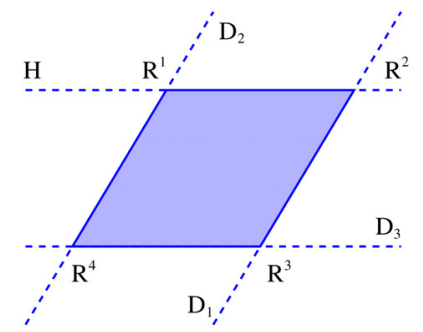

Clearly the elementary contractions associated with $R^{1}$ and $R^{2}$ are the the blowdowns of $E_{1}$ and $E_{2}$. The elementary contraction associated with $R^{3}$ is divisorial, and its exceptional locus is the strict transform of the join $J\left(\Lambda_{1}, Q_{2}\right)$, which is a divisor linearly equivalent to $2 \mathcal{H}-2 E_{1}-E_{2}$. Finally, the contraction associated with $R^{4}$ contracts the strict transform of $\Lambda_{2}$. Hence, if $\operatorname{dim} \Lambda_{2}=n-1$ this contraction is divisorial, otherwise it is small.

Description of the families of rational curves. In this example the family $V$ of cubics is the family of deformations of the strict transform of a general line of $\mathbb{P}^{n}$; the set $\mathcal{B}^{\prime}$ consists of two pairs, $\left(\mathcal{L}^{1}, C^{1}\right)$ and $\left(\mathcal{L}^{2}, C^{2}\right)$ : the families $\mathcal{L}^{i}$ are the families of lines contracted by the blow-down, the family $C^{1}$ is the family of strict transforms of lines in $\mathbb{P}^{n}$ meeting $\Lambda_{1}$ at one point and the family $C^{2}$ is the family of strict transforms of lines in $\mathbb{P}^{n}$ meeting $Q_{1}$ at one point. 
Curves parametrized by $C^{1}$ degenerate into the strict transform of a line meeting $\Lambda_{1}$ and $Q_{1}$ and a line contracted by $\varepsilon_{2}$, while curves parametrized by $C^{2}$ degenerate in two possible ways: either as a line contracted by $\varepsilon_{2}$ and the strict transform of a line contained in $\Lambda_{2}$ or as a line contracted by $\varepsilon_{1}$ and the strict transform of a line meeting both $\Lambda_{1}$ and $Q_{1}$.

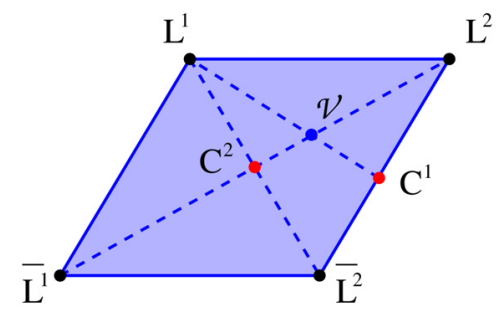

Example 8.3. $(X, H) \simeq\left(B l_{Q_{1}, Q_{2}}\left(\mathbb{P}^{n}\right), 3 \mathcal{H}-E_{1}-E_{2}\right)$, where $B l_{Q_{1}, Q_{2}}\left(\mathbb{P}^{n}\right)$ is the blow-up of $\mathbb{P}^{n}$ along two smooth quadrics $Q_{1} \subset \Lambda_{1} \simeq \mathbb{P}^{\frac{n}{2}}$ and $Q_{2} \subset \Lambda_{2} \simeq \mathbb{P}^{\frac{n}{2}}$ such that

$$
Q_{1} \cap Q_{2}=\emptyset, \quad \operatorname{dim} \Lambda_{1} \cap \Lambda_{2}=0, \quad \operatorname{dim} Q_{1}=\operatorname{dim} Q_{2}=\frac{n}{2}-1,
$$

$E_{1}, E_{2}$ are the exceptional divisors of $\pi$ and $\mathcal{H}=\pi^{*} \mathcal{O}_{\mathbb{P}^{n}}(1)$. Denote by

- $R^{i}$ the extremal ray corresponding to the contraction of $E_{i}$;

- $\varepsilon_{i}$ the contraction associated with $R^{i}$;

- $\ell_{i}$ a minimal curve whose numerical class is in $R^{i}$;

- $\bar{\ell}_{i}$ a curve which is the strict transform of a line contained in $\Lambda_{i}$;

- $D_{i}=2 \mathcal{H}-E_{i}$;

- $D_{3}=2 \mathcal{H}-E_{2}-E_{1}$.

As in Example 8.2 we can show that $\mathrm{NE}(X)$ is the intersection of the halfspaces determined by the nef divisors $\mathcal{H}, D_{1}, D_{2}, D_{3}$, that is spanned by four rays,

$$
R^{1}=\mathbb{R}_{+}\left[\ell_{1}\right], \quad R^{2}=\mathbb{R}_{+}\left[\ell_{2}\right], \quad R^{3}=\mathbb{R}_{+}\left[\bar{\ell}_{1}\right], \quad R^{4}=\mathbb{R}_{+}\left[\bar{\ell}_{2}\right],
$$

and that the position of these rays is as in the next figure, which shows a cross section of $\mathrm{NE}(X)$.

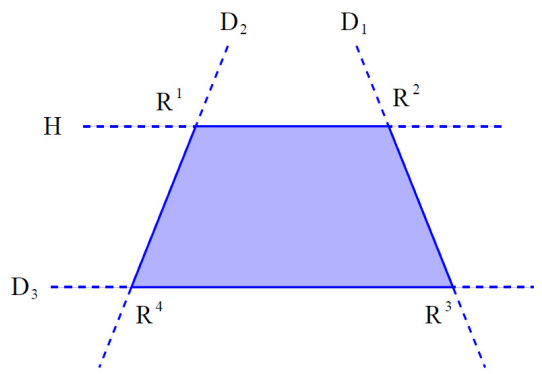

Clearly the elementary contractions associated with $R^{1}$ and $R^{2}$ are the the blowdowns of $E_{1}$ and $E_{2}$. The elementary contractions associated with $R^{3}$ and with $R^{4}$ 
are small with exceptional loci of dimension $\frac{n}{2}$ which are the strict transforms of the linear spaces $\Lambda_{i}$.

Let us devote a few words to the contraction of the face $\sigma=\left\langle R^{3}, R^{4}\right\rangle$. Let $P$ be the intersection point of $\Lambda_{1}$ and $\Lambda_{2}$, and let $\Sigma$ be a 2-plane passing through $P$ and meeting $\Lambda_{1}$ and $\Lambda_{2}$ in two lines, $l_{1}$ and $l_{2}$. It is not difficult to see that for a general point of $\mathbb{P}^{n}$ there is exactly one such 2 -plane.

For a general point $Q$ of $\Sigma$ there is a conic passing through $Q$ and through the (four) points of intersection of $\Sigma$ with $Q_{1}$ and $Q_{2}$; denote by $\gamma$ this conic. Since $\gamma$ meets both $Q_{1}$ and $Q_{2}$ in two points we have $E_{1} \cdot \gamma=E_{2} \cdot \gamma=2$.

The contraction $\varphi: X \rightarrow Y$ of $\sigma$ is supported by $D_{3}=2 \mathcal{H}-E_{1}-E_{2}$. Hence, it contracts $\gamma$; it follows that $\varphi$ is of fiber type. The restriction of $D_{3}$ to $E_{1}$ is big, hence $\operatorname{dim} Y=n-1$.

Therefore $\varphi$ is a conic bundle; the divisor of reducible conics is the strict transform of the join $J\left(Q_{1}, Q_{2}\right)$, and there is one special fiber of dimension $n-2$ consisting of two irreducible components which are projective spaces meeting at a point, namely the strict transforms of $\Lambda_{1}$ and $\Lambda_{2}$.

Description of the families of rational curves. In this example the family $V$ of cubics is the family of deformations of the strict transform of a general line of $\mathbb{P}^{n}$; the set $\mathcal{B}^{\prime}$ consists of two pairs, $\left(\mathcal{L}^{1}, C^{1}\right)$ and $\left(\mathcal{L}^{2}, C^{2}\right)$ : the families $\mathcal{L}^{i}$ are the families of lines contracted by the blow-down, while the families $C^{i}$ are the families of strict transforms of lines in $\mathbb{P}^{n}$ meeting $Q^{i}$ at one point.

Curves in $C^{i}$ degenerate in two possible ways: either as a line contracted by $\varepsilon_{i}$ and the strict transform of a line contained in $\Lambda_{i}$ or as a line contracted by $\varepsilon_{j}$ $(i \neq j)$ and the strict transform of a line meeting both $Q_{1}$ and $Q_{2}$. These last curves are numerically proportional to the conics meeting both $Q_{1}$ and $Q_{2}$ in two points.

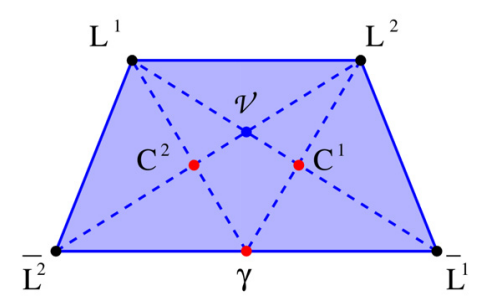

Acknowledgements. We would like to thank Francesco Russo and Massimiliano Mella for many useful comments and suggestions.

Note. This work grew out of a part of the second author's Ph.D. thesis [16] at the Department of Mathematics of the University of Trento.

\section{References}

[1] Andreatta, M., Chierici, E. And Occhetta, G.: Generalized Mukai conjecture for special Fano varieties. Cent. Eur. J. Math., 2 (2004), no. 2, 272-293.

[2] Andreatta, M. And Wiśniewski, J. A.: A note on nonvanishing and applications. Duke Math. J. 72 (1993), no. 3, 739-755. 
[3] Beltrametti, M. C. and Sommese, A. J.: The adjunction theory of complex projective varieties. de Gruyter Expositions in Mathematics, 16. Walter de Gruyter, Berlin, 1995.

[4] Beltrametti, M. C., Sommese, A. J. And Wiśniewski, J. A.: Results on varieties with many lines and their applications to adjunction theory. In Complex algebraic varieties (Bayreuth, 1990), 16-38. Lecture Notes in Math., 1507. Springer, Berlin, 1992.

[5] Bonavero, L., Casagrande, C. and Druel, S.: On covering and quasi-unsplit families of curves. J. Eur. Math. Soc. 9 (2007), no. 1, 45-57.

[6] Chierici, E. And Occhetta, G.: Fano fivefolds of index two with blow-up structure. Tohoku Math. J. (2) 60 (2008), no. 4, 471-498.

[7] Debarre, O.: Higher-dimensional algebraic geometry. Universitext. SpringerVerlag, New York, 2001.

[8] Harris, J.: Algebraic geometry. A first course. Graduate Texts in Mathematics, 133. Springer-Verlag, New York, 1992

[9] Ionescu, P.: Generalized adjunction and applications. Math. Proc. Cambridge Philos. Soc. 99 (1986), no. 3, 457-472.

[10] Ionescu, P. And Russo, F.: Conic-connected manifolds. J. Reine Angew. Math. 644 (2010), 145-158.

[11] Kebekus, S.: Characterizing the projective space after Cho, Miyaoka and ShepherdBarron. In Complex geometry (Göttingen, 2000), 147-155. Springer, Berlin, 2002.

[12] Kebekus, S.: Families of singular rational curves. J. Algebraic Geom. 11 (2002), no. 2, 245-256.

[13] Kollár, J.: Rational curves on algebraic varieties. Results in Mathematics and Related Areas. 3rd Series. A Series of Modern Surveys in Mathematics, 32. SpringerVerlag, Berlin, 1996.

[14] Miyaoka, Y.: Numerical characterisations of hyperquadrics. In Complex analysis in several variables - Memorial Conference of Kiyoshi Oka's Centennial Birthday, 209-235. Adv. Stud. Pure Math., 42. Math. Soc. Japan, Tokyo, 2004.

[15] Occhetta, G. and Paterno, V.: Rationally cubic connected manifolds I: manifolds covered by lines. J. Math. Soc. Japan 64 (2012), no. 3, 941-967.

[16] Paterno, V.: Special rationally connected manifolds. PhD thesis. Available at http://eprints-phd.biblio.unitn.it/159/.

[17] Wiśniewski, J.A.: On contractions of extremal rays of Fano manifolds. J. Reine Angew. Math., 417 (1991), 141-157.

Received September 20, 2010; revised April 21, 2011.

Gianluca Occhetta and Valentina Paterno: Dipartimento di Matematica, via Sommarive 14, 38123 Povo (TN), Italy.

E-mail: gianluca.occhetta@unitn.it, valentina.paterno@gmail.com

Partially supported by MIUR (PRIN project: Proprietà geometriche delle varietà reali e complesse). 